\title{
O papel da equidade no planejamento de transporte coletivo urbano no Brasil
}

\author{
Thiago Guimarães ${ }^{1}$, Karen Lucas ${ }^{2}$ \\ 1Institute for Transport Studies, University of Leeds, Reino Unido, th.guimaraes@gmail.com \\ 2Institute for Transport Studies, University of Leeds, Reino Unido, K.Lucas@leeds.ac.uk
}

\section{Recebido:}

31 de maio de 2018

Aceito para publicação:

01 de maio de 2019

Publicado:

31 de dezembro de 2019

Editor de área:

Cira Souza Pitombo

\section{Palavras-chaves:}

Equidade,

Transporte coletivo,

Planejamento de transporte,

Mobilidade urbana sustentável.

\section{Keywords:}

Equity,

Public transport,

Transport planning,

Sustainable urban mobility.

DOI:10.14295/transportes.v27i4.1709

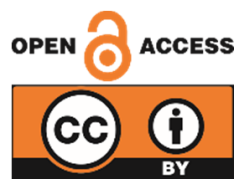

\begin{abstract}
RESUMO
Embora a noção de equidade seja considerada primordial no emergente paradigma da mobilidade urbana sustentável, o planejamento da mobilidade tem sido acusado de contribuir para a produção e a consolidação de desigualdades sociais e espaciais relacionadas à provisão e organização do transporte coletivo urbano, principalmente no contexto de países em desenvolvimento. Este artigo investiga em que medida planejadores atuantes em cidades brasileiras estão cientes de iniquidades relacionadas ao transporte e a forma como compreendem o papel do transporte em relação a objetivos sociais. Diferentemente do que a literatura sugere, resultados da survey conduzida para este estudo indicam que, no Brasil, planejadores de transportes reconhecem certas associações entre privação socioeconômica e deficiências na oferta de transporte coletivo urbano, mas não necessariamente identificam no processo de planejamento fatores que levam à produção de desigualdades. Tampouco compreendem a ideia de equidade social como central no paradigma de mobilidade urbana sustentável.
\end{abstract}

\begin{abstract}
The notion of equity is primordial in the emerging paradigm of sustainable urban mobility. However, especially in the developing context, transport planning has been blamed for producing social and spatial inequities, related to lacks of provision and organisational inadequacies of urban collective transport. This article investigates the extent to which practitioners in Brazilian cities are aware of iniquities associated with transport and how they understand the interrelationships between transport and social issues. Differently from what the academic literature suggests, results of the survey conducted for this study indicate that transport planners in Brazil recognise associations between socioeconomic deprivation and deficiencies in the provision of urban public transport, but they do not necessarily hold the planning process responsible for the production of these inequalities, nor do they understand the idea of social equity as a pillar of the sustainable urban mobility paradigm.
\end{abstract}

\section{INTRODUÇÃO}

Apesar de controverso, o conceito de equidade é fundamental para a formulação de políticas de transportes e para o planejamento de transportes. 0 conceito se faz presente em vários objetivos estratégicos, declarados ou implícitos, de políticas públicas de transportes e projetos de mobilidade urbana tais como: a promoção de um desenvolvimento urbano equilibrado; a criação de oportunidades equivalentes de acesso para todos os grupos populacionais; a provisão de acesso a equipamentos públicos, serviços e oportunidades; e a satisfação de uma gama de necessidades básicas, em particular de grupos sociais desfavorecidos. Mais recentemente, preocupações com justiça social permearam a evolução do debate em torno da mobilidade sustentável. Neste paradigma, a equidade é mencionada como um princípio-chave (Gudmundsson et al., 2016). 
No entanto, o planejamento de transportes urbanos tem sido acusado de não contribuir para fomentar a equidade e a melhoria da qualidade de vida de toda a população, por exemplo quando não contribui para reduzir as disparidades relativas à acessibilidade aos sistemas de transportes e às oportunidades (Banister, 2002; Jones e Lucas, 2012; Manaugh et al., 2015). Pelo contrário, tem sido fartamente documentado o modo como intervenções planejadas nos sistemas de transportes podem não só deixar de satisfazer necessidades de segmentos sociais desfavorecidos como, em muitos casos, prejudicar populações em situação socioeconômica vulnerável, gerando e reproduzindo desigualdades. Por exemplo, em consequência direta de um modelo de planejamento de transportes que molda ambientes urbanos em benefício de grupos sociais com acesso a automóveis e negligencia investimentos em modos coletivos e não motorizados de transporte, indivíduos de baixa renda podem ser forçados a gastar substantiva parcela de seus rendimentos com mobilidade, estar expostos a níveis desproporcionalmente altos de ruído e poluição, bem como ao risco de ferimentos e fatalidades relacionados ao tráfego, e podem sofrer com o "efeito-barreira" criado pela abertura de grandes vias nos bairros onde residem (Jones e Lucas, 2012; Sanchez et al., 2003; Soja, 2010).

No Brasil, a maioria das cidades é caracterizada por marcante segregação espacial, acentuada disparidade nos níveis de acessibilidade a atividades e oportunidades distribuídas no território urbano e graves deficiências na provisão de infraestrutura, inclusive de transportes (Portugal, 2017). Mesmo assim, substanciais investimentos de transporte em cidades brasileiras têm privilegiado classes de alta renda, como o desenvolvimento da rede de metrô de São Paulo (Villaça e Zioni, 2010). Em cidades brasileiras, o transporte público pode ser inexistente, considerado muito caro ou até mesmo inútil na perspectiva dos moradores de comunidades de baixa renda (Maia et al., 2016). Em comparação internacional, algumas cidades brasileiras foram identificadas como aquelas em que o transporte público é, em termos financeiros, o menos acessível aos segmentos mais pobres da população, que, dessa forma, tem sua participação em atividades severamente limitada (Carruthers et al., 2005). Apesar de a legislação estabelecer a redução das desigualdades e promover a inclusão social como objetivos estratégicos das políticas urbanas, a política de transportes no Brasil ainda é considerada socialmente seletiva e altamente excludente (Vasconcellos, 2014). Como em outros lugares do Sul Global (Dimitriou, 1992), argumenta-se que o planejamento de transportes praticado no Brasil é insensível às questões sociais, tendendo a favorecer grupos de maior status social (Vasconcellos, 2001).

Alguns estudiosos exploraram teorias político-filosóficas para apontar maneiras de incorporar princípios de justiça social nos processos de planejamento e tomada de decisão (Martens, 2012, 2017; Pereira, Schwanen, et al., 2017). Outros autores discutiram em que medida as considerações de equidade social têm sido incluídas em planos de transporte. Nos planos produzidos para as principais áreas metropolitanas da América do Norte, preocupações com a justiça social estão vagamente ancoradas e são raramente traduzidas em objetivos e indicadores (Manaugh et al., 2015). Com a possível exceção de alguns trabalhos do início dos anos 1990 nos Estados Unidos e no Reino Unido (Dimitriou, 1992; Hay e Trinder, 1991), pesquisas sobre como os planejadores entendem a relação entre transporte e equidade social através da aferição de seus valores, perspectivas e experiências ainda são raros. Alguns estudos tentaram apresentar possíveis fatores para explicar como e por que o planejamento de transportes e a formulação de políticas podem fomentar as desigualdades nos países em desenvolvimento (Dimitriou, 1992; Vasconcellos, 2001). Estes estudos são revisados mais adiante neste artigo. 
0 presente artigo tem como perspectiva ampliar a compreensão do papel da equidade no planejamento do transporte no Brasil por meio da aferição de opiniões e pontos de vista de profissionais. 0 estudo explora, especificamente, a interface entre equidade e o planejamento de sistemas de transporte público urbano, em particular, por ônibus, que é o modo coletivo mais comum nas cidades brasileiras, utilizado por diversos grupos sociais e cuja capilaridade lhe permite atender bairros em um amplo espectro de condições socioeconômicas.

0 estudo é guiado pelas seguintes questões:

1. De quais formas de desigualdades sociais e espaciais relacionadas ao transporte urbano coletivo planejadores brasileiros têm ciência?

2. Quais os pontos de vista dos profissionais sobre o papel do planejamento de transportes coletivos em relação à equidade social?

3. Qual prioridade planejadores dão a estratégias voltadas ao transporte público que tendem a promover a equidade?

4. Os planejadores associam equidade a projetos de mobilidade urbana sustentável?

0 artigo é estruturado em cinco seções, incluindo esta introdução. A Seção 2, correspondente à revisão de literatura, oferece um amplo panorama sobre as diversas perspectivas de equidade adotadas em estudos de transporte e também discute a relação entre equidade nos transportes e mobilidade sustentável. A Seção 3 fornece detalhes sobre o método empregado nesta pesquisa para aferir os pontos de vista dos profissionais em particular no que se refere a intervenções estratégicas no sistema de transporte coletivo. A Seção 4 apresenta os resultados. A Seção 5 discute e sintetiza os principais resultados desse trabalho.

\section{REVISÃO DA LITERATURA}

\subsection{Narrativas plurais sobre equidade em transportes}

O planejamento e a política de transporte envolvem reflexões sobre equidade em termos proceduais e substantivos relacionados ao desenho de sistemas de transporte para distintos grupos de usuários, a alocação de recursos e a definição de prioridades de investimentos em transportes e a perseguição do objetivo estratégico de garantir mobilidade a todos os indivíduos para que consigam atender às suas necessidades básicas de participação em atividades. Segundo Banister (2002), uma das funções centrais do planejamento consiste em garantir uma distribuição justa dos recursos, de modo que todas as pessoas tenham acesso aos equipamentos de que necessitam. Embora equidade em transportes não seja um assunto novo - sua origem está relacionada ao movimento pelos direitos civis nos anos 1960 nos Estados Unidos (Dimitriou, 1992; Sanchez, 2008) - é uma noção bastante abrangente e que interage com temas desenvolvidos em outras disciplinas (por exemplo, vulnerabilidade social e segregação residencial). Esta seção fornece um panorama dos principais aspectos relacionados ao debate sobre equidade em transportes.

Embora acadêmicos tenham reconhecido há muito tempo que diferentes princípios de equidade possam guiar o planejamento e a avaliação de transportes para garantir a justiça das intervenções propostas (Thomopoulos et al., 2009; Trinder et al., 1991; van Wee, 2011), a maioria dos estudos em transporte tem se mantido evasivo sobre um posicionamento teórico em relação à justiça social (Martens, 2017; Martens e Lucas, 2018). Avanços recentes neste campo incluem a proposição de abordagens analíticas explicitamente fundamentadas em enquadramentos político-filosóficos de justiça social. Embora posturas igualitárias e suficientaristas também 
tenham sido adotadas (Lucas et al., 2016; van Wee, 2011), as perspectivas liberais de justiça por John Rawls (e.g. Martens, 2017; Pereira et al., 2017) e a abordagem das "capabilities" proposta por Amartya Sen e Martha Nussbaum (Hananel e Berechman, 2016; Nahmias-Biran et al., 2017; Ryan et al., 2015; Smith et al., 2012) têm sido preferidas por diversos autores. Em comum, esses estudos contribuem para ilustrar aspectos de desigualdades sociais relacionadas aos transportes a partir de uma conceituação filosófica de justiça geralmente divergente do utilitarismo (o corpo teórico que fundamenta as técnicas de planejamento e avaliação de transportes convencionais).

Diferentes abordagens para a equidade do transporte também podem derivar de entendimentos distintos dos recursos relacionados ao transporte que devem ser objeto de uma distribuição equitativa. Uma maneira bastante difundida de abordar a equidade no transporte baseiase nas diferenças observáveis no comportamento relativo a viagens de diferentes segmentos sociais e no fato de que as desigualdades sociais estão associadas a diferentes níveis de mobilidade (Ohnmacht et al., 2009). Os indicadores de mobilidade, como o número de viagens por unidade de tempo, os tempos médios ou as distâncias de viagem geralmente diferem entre grupos segmentados por renda, modo de transporte utilizado (por exemplo, motoristas de automóvel particular vis-à-vis usuários de transporte público), deficiências físicas ou mobilidade reduzida, ou gênero, que consiste em uma dimensão transversal (Uteng, 2008, 2009). Um posicionamento crítico perante tais desigualdades também nutriu a ideia de mobilidade como um direito básico do cidadão (Cresswell, 2006).

Quando o foco recai sobre o desenho das instituições que devem garantir que todos os membros da sociedade tenham o mesmo direito e possam participar efetivamente do processo de planejamento ou influenciar as decisões políticas, discursos sobre equidade em transporte se relacionam intimamente com justiça processual. Ampliar o engajamento dos cidadãos, em particular de grupos sociais cuja voz tem sido sistematicamente ignorada por práticas de planejamento racional e tecnocrático, tem sido considerada uma maneira de influenciar agendas políticas para um transporte mais equitativo (Bickerstaff et al., 2002; Elvy, 2014; Fouracre et al., 2006; Hodgson e Turner, 2003; Sagaris, 2010; Vigar, 2006, 2017).

Outros estudos concentram-se em avaliar a equidade na distribuição espacial das infraestruturas e serviços, bem como a conectividade dos transportes. Atingir a meta política de condições de vida equivalentes para cidadãos residentes em áreas distintas de um território pode depender da localização e distribuição de vias, pontos de ônibus, estações de metrô e garantir a oferta de transporte de qualidade em diferentes áreas (Caggiani et al., 2017; Santos et al., 2008). Relacionadas a essas questões, preocupações com a acessibilidade dos transportes - aí incluídas as questões da posse forçada de automóveis ("forced car ownership") e a pobreza energética ("fuel poverty") (Curl et al., 2018; Currie e Delbosc, 2009; Mattioli, 2015, 2017; Mattioli et al., 2017, 2016) - ganharam atenção crescente no bojo das recentes oscilações do preço do combustível e com base no reconhecimento de que a tarifa do transporte público pode consistir em uma barreira severa de acesso para uma parcela expressiva da população (Falavigna e Hernandez, 2016; Guzman e Oviedo, 2018). A cobertura espacial de redes de transporte e a extensão de esquemas como proibições de estacionamento e cobranças pela utilização do sistema viário também podem refletir preocupações de equidade (Levinson, 2010; Li et al., 2018; Liu et al., 2019). 
Um grande número de pesquisas concentrou-se na distribuição de impactos relacionados ao transporte entre diferentes segmentos populacionais, em particular nas externalidades negativas associadas ao uso de modos de transporte motorizados, como a poluição do ar (Clark et al., 2017; Houston et al., 2004; Milojevic et al., 2017; Sider et al., 2015), o ruído do tráfego motorizado (Houston et al., 2004; Milojevic et al., 2017; Mueller et al., 2018; Sider et al., 2015; Tonne et al., 2018) e as mortes e ferimentos causados por "acidentes de trânsito" (Morency et al., 2012). Por exemplo, estudos sobre justiça ambiental ("environmental justice"), principalmente realizados no contexto estadounidense, em geral utilizam sistemas de informação geográfica para avaliar se grupos étnicos e de baixa renda estão desproporcionalmente expostos a emissões relacionadas ao transporte e outras fontes de impactos deletérios para a saúde humana (Bullard e Johnson, 1997; Chakraborty et al., 2011, 1999; Gaffron, 2012; Rowangould et al., 2016).

Conceitualmente, o debate acadêmico sobre os impactos sociais do transporte tem enfatizado a ampla gama de recursos relacionados ao transporte que são relevantes para o bem-estar humano e que vão além dos fatores predominantes em avaliações de projetos de transporte (por exemplo, economia de tempo de viagem) (Forkenbrock et al., 2001; Geurs et al., 2009; Jones e Lucas, 2012; Markovich e Lucas, 2011). Em particular, proliferaram nas duas últimas décadas estudos que analisam a distribuição da acessibilidade a atividades essenciais (como emprego, educação e serviços públicos) entre diferentes áreas e grupos sociais (Martens e Lucas, 2018). Estudos abordando a acessibilidade em relação à equidade social baseiam-se amplamente em resultados de pesquisas sobre a exclusão social relacionada a transportes, que contribuíram para identificar relações de causa e efeito entre deficiências nos sistemas de transporte e múltiplas formas de desvantagem social (Cass et al., 2005; Church et al., 2000; Farrington e Farrington, 2005; Hine e Mitchell, 2001; Kenyon et al., 2002; Lucas, 2004). Conforme esta literatura, grupos socialmente desfavorecidos incluem pessoas com baixa renda domiciliar, desempregados, mulheres, pais solteiros, idosos, pessoas com deficiência, crianças e jovens, certas minorias étnicas e pessoas que vivem em áreas com baixa densidade populacional (Hine e Grieco, 2003; Lucas, 2004, 2012; Lucas et al., 2016).

Embora todas essas correntes tenham explorado evidências no Norte Global, outro ramo da literatura tenta situar equidade em transporte em países em desenvolvimento, reconhecendo que, neste contexto, questões sociais ligadas ao transporte estão entrelaçadas com processos específicos de rápido crescimento populacional, expansão urbana descontrolada, segregação residencial, fracas estruturas de governança urbana, regulamentação deficiente dos serviços de transportes, presença de assentamentos não planejados e ilegais, e uma alta parcela da população vivendo em situação de pobreza ou miséria. Tipicamente, esses estudos relatam grandes disparidades nos tempos de viagem, exposição a riscos para a saúde humana relacionados ao trânsito e a participação dos gastos com transporte nos orçamentos domésticos nos diferentes segmentos sociais (Vasconcellos, 2001, 2018). Pesquisas recentes avaliaram como a acessibilidade é distribuída desigualmente entre a população, por exemplo, nas cidades da América Latina (Guzman et al., 2017; Jaramillo et al., 2012; Oviedo Hernandez e Titheridge, 2016; Pereira et al., 2017). Esses estudos também fornecem ideias valiosas para contrabalançar o atual domínio das narrativas sobre a equidade em transporte produzido no Norte Global (Dimitriou, 1992; Falavigna et al., 2017a, 2017b; Godard, 2011; Vasconcellos, 2001). 


\subsection{Equidade e mobilidade sustentável}

Para alguns autores, a noção de sustentabilidade está no cerne da alternativa mais promissora de enquadrar conceitualmente os problemas contemporâneos de transporte e uma forma de superá-los. 0 paradigma da mobilidade sustentável é mais focado na acessibilidade potencial e realizada do que no incremento da mobilidade e na velocidade dos deslocamentos (Banister, 2008; Bertolini, 2012; Bertolini et al., 2008; Hull, 2008; Jones, 2014; Litman, 2013).

No entanto, há controvérsias em relação à medida em que o paradigma da mobilidade sustentável está interligado com preocupações de equidade. Alguns autores enfatizam que a equidade constitui um dos três fundamentos da sustentabilidade, juntamente com as dimensões econômica e ambiental, e que essa tríade também sustenta o paradigma da mobilidade sustentável (Gudmundsson et al., 2016). Documentos políticos que representaram importantes contribuições para a compreensão atual da mobilidade sustentável fazem referência explícita ao papel social dos transportes, à criação de oportunidades para as regiões periféricas e ao aumento da acessibilidade como objetivo central na política de transportes (Gudmundsson et al., 2016). Também se argumenta que, dentro do paradigma da sustentabilidade, as estratégias de transporte tendem a ser avaliadas levando-se em conta os impactos sobre a equidade social, juntamente com a eficiência e os indicadores ambientais (Litman, 2013).

Outros estudiosos, no entanto, afirmam que o elo entre sustentabilidade e equidade (ou inclusão social) é mais uma questão de retórica acadêmica ou política do que um fato em si. Eles enfatizam que o planejamento de transportes e os instrumentos de avaliação de investimentos no setor, mesmo quando rotulados "sustentáveis", ainda são demasiadamente focados nos ganhos de eficiência no setor de transportes. Ou seja, a mobilidade sustentável não tem como foco a equidade. Por isso, esses autores geralmente privilegiam terminologias mais específicas, como "sustentabilidade social" (Boschmann e Kwan, 2008; Grieco, 2015; Lucas e Stanley, 2013; Manzi et al., 2010). Tais visões céticas estão alinhadas com os resultados de estudos recentes como os de Arsenio et al. (2016), que concluíram que a equidade social não foi abordada de forma adequada na maioria dos 40 planos de mobilidade urbana sustentável, elaborados para cidades portuguesas, apesar da presença de preocupações pontuais com a melhoria da acessibilidade de idosos e de pessoas com deficiência.

Acadêmicos têm considerado a equidade social um princípio particularmente eminente nas narrativas sobre mobilidade sustentável contextualizadas no Sul Global. Para Banister (2005), o desenvolvimento urbano sustentável apresenta-se como um conceito social no Sul Global em oposição ao Norte Global, onde é tratado como um conceito ambiental. Outros autores afirmam que os planos de mobilidade desenvolvidos para cidades do Sul Global geralmente reconhecem o papel potencial do transporte no alívio da pobreza e incluem a equidade social como uma questão a ser abordada (Bocarejo e Oviedo, 2012).

No Brasil, o discurso sobre "mobilidade sustentável" foi alavancado por meio de iniciativas de capacitação e do arcabouço legal recentemente estabelecido pelo governo federal através da Política Nacional de Mobilidade Urbana, que definiu equidade social, justa distribuição de benefícios e ônus decorrentes de diferentes modos e serviços de transporte, equidade no acesso ao transporte público, acessibilidade universal e equidade no consumo de espaço como princípios norteadores dos planos locais de transporte (Miranda e Rodrigues da Silva, 2012; Portugal, 2017; Rodrigues da Silva et al., 2008). No entanto, a tradução de tais aspirações normativas em intervenções concretas capazes efetivamente de combater desigualdades 
continua sendo um desafio no contexto brasileiro. Argumentamos, neste artigo, que pelo menos algumas das razões para isso podem ser encontradas nas opiniões, percepções e atitudes da comunidade profissional sobre o papel do transporte em relação às desigualdades sociais.

\section{MÉTODO}

Uma pesquisa do tipo survey online e de corte transversal foi o principal método de coleta de dados utilizado para avaliar como os profissionais brasileiros de planejamento de transportes percebem e justificam a existência de desigualdades no transporte, o papel da equidade em suas práticas e como eles relacionam equidade ao conceito de mobilidade sustentável. Surveys eletrônicas têm a vantagem de atingir participantes espacialmente dispersos a custos relativamente baixos. 0 público-alvo pode ser alcançado sem nenhum viés inerente, uma vez que computadores com acesso à Internet consistem em um recurso geralmente acessível a profissionais de planejamento de transporte.

O questionário consistiu em 37 questões padronizadas - uma combinação de perguntas fechadas (escalas ordenadas numericamente, escolha múltipla e escolha ordenada) e abertas. Solicitou-se aos participantes expressar o nível de concordância com relação a uma série de afirmações sobre a existência de desigualdades no transporte, opinar sobre a função social do transporte público urbano em face a desigualdades e mencionar projetos de transporte que consistem em boas práticas com respeito a seus impactos sociais e distributivos. Os entrevistados também elencaram medidas prioritárias de um conjunto de possíveis estratégias para o desenvolvimento do transporte coletivo no contexto da cidade em que desenvolvem suas atividades profissionais. As questões se referiam a serviços de ônibus, dentro do contexto geográfico conhecido pelos participantes.

Graus de concordância puderam ser expressos em uma escala Likert variando entre 1 (completa discordância) e 6 (completa concordância). 0 formato das opções de resposta com um número par de alternativas excluiu intencionalmente o ponto médio para evitar a tendência para a neutralidade, usualmente exibida quando participantes deparam-se com questões complexas. Esta opção metodológica também se baseou em um posicionamento normativo dos pesquisadores de que é improvável que os participantes consigam manter-se absolutamente neutros em relação às afirmações contidas nesta pesquisa. A opção de resposta "Eu não sei" estava disponível para todas as questões.

Para melhor atingir o público-alvo, a pesquisa foi anunciada entre os participantes de um curso à distância sobre gestão da mobilidade urbana, coordenado pela Associação Nacional de Transportes Públicos (ANTP) e patrocinado pelo Banco Mundial, bem como em fóruns temáticos especializados em redes sociais. Partindo da premissa de que inscritos no curso estariam propensos a compartilhar informações sobre suas experiências (este era um requisito para participar do curso), a estratégia de recrutamento pode ter ampliado o acesso a entrevistados com maior potencial de envolvimento, aumentando potencialmente a validade dos resultados da pesquisa. Além disso, como forma de incentivar a participação, para cada grupo de 50 respondentes foi anunciado o sorteio de um vale-presente que poderia ser utilizado em uma rede de comércio eletrônico.

O questionário foi implementado com a ferramenta SosciSurvey e a coleta de dados ocorreu entre 15 e 29 de outubro de 2015. Ao todo, 94 profissionais de mobilidade urbana completaram o questionário e nove abandonaram a pesquisa sem finalizá-la, tendo suas contribuições desconsideradas. 
Dado o pequeno tamanho de sua amostra em relação ao universo de profissionais de mobilidade urbana no Brasil e seu desenho metodológico (amostragem por oportunidade), a pesquisa não pode ser considerada estatisticamente representativa. No entanto, acredita-se que as informações coletadas na pesquisa são válidas e refletem fielmente as posições dos entrevistados, já que $77,7 \%$ afirmaram ter entendido todas as perguntas e outros $20,2 \%$ entenderam facilmente a maioria das questões da pesquisa. Além disso, o tempo investido para participar da pesquisa (aproximadamente 20 minutos, em média) foi considerado razoável pela maioria $(83,0 \%)$. Isso indica que a qualidade das respostas não deve ter sido afetada pelo efeito-fadiga, que surge quando participantes são expostos a um processo de entrevista percebido como demasiadamente longo. A grande maioria dos entrevistados considera ter um bom conhecimento do sistema de transporte público nas cidades onde trabalham, o que também subsidia a validade dos resultados da pesquisa.

\section{RESULTADOS}

\subsection{Caracterização dos respondentes}

Participaram da survey profissionais de transporte de 16 unidades federativas, com predomínio de participantes dos estados de São Paulo (29,8\%), Minas Gerais (22,3\%) e do Distrito Federal (16,0\%). 88,3\% dos participantes atuam em cidades com população superior a 250 mil habitantes. Municípios desse porte caracterizam-se por uma parcela significativa dos deslocamentos diários realizados por transporte coletivo, maior intensidade dos problemas de circulação e, no plano institucional, contam com estruturas dedicadas à gestão e ao planejamento da mobilidade urbana (Ministério das Cidades, 2015).

Os resultados mostram que a maioria dos participantes são do sexo masculino (62,8\%), bran$\cos (71,3 \%)$, vivendo e trabalhando em áreas urbanas populosas. 51\% dos entrevistados têm entre 25 e 40 anos. A remuneração do trabalho média estimada é R \$ 5,7 mil (o que correspondia a pouco mais de 7 salários mínimos em valores da época). Os entrevistados geralmente completaram o ensino superior: 68,3\% possuem graduação, 18,1\% mestrado e 6,4\% título de doutorado. As carreiras universitárias mais frequentes são engenharia, arquitetura e administração. Três entre quatro respondentes trabalham para o setor público (como governos locais, autoridades públicas e universidades).

\subsection{Reconhecimento de desigualdades em transporte}

Os participantes da pesquisa foram confrontados com declarações sobre a existência de desigualdades relacionadas ao transporte nas cidades onde trabalham. As declarações eram:

- Todos os bairros são bem servidos por ônibus.

- Regiões mais pobres da cidade têm serviços de transporte coletivo de menor qualidade do que regiões mais ricas.

- As pessoas mais pobres demoram mais tempo para chegar ao local de trabalho do que as mais ricas.

- A tarifa de ônibus pode ser paga por todos aqueles que precisam usar o serviço.

Conforme exibido na Figura 1, a maioria dos entrevistados demonstra ter ciência da existência da relação entre desigualdades espaciais e sociais, e o transporte público. Aproximadamente dois terços dos participantes percebem que o transporte coletivo não funciona adequadamente pelo menos em alguns bairros. Em particular, reconhecem que as áreas de baixa renda estão 
conectadas por serviços de transporte de menor qualidade em comparação com áreas mais ricas (70,2\% dos entrevistados expressam concordância com esta declaração e, entre elas, 30,9\% concordam totalmente). Além disso, os participantes acreditam que os custos e o tempo de viagem podem representar barreiras significativas para a utilização de serviços de transporte. 91,5\% percebem que grupos sociais de baixa renda gastam mais tempo para deslocamentos ao trabalho do que os mais ricos. Sete em cada dez participantes entendem que alguns usuários, mesmo dependendo do transporte público, possam ser impedidos de utilizá-lo em função do custo da tarifa.

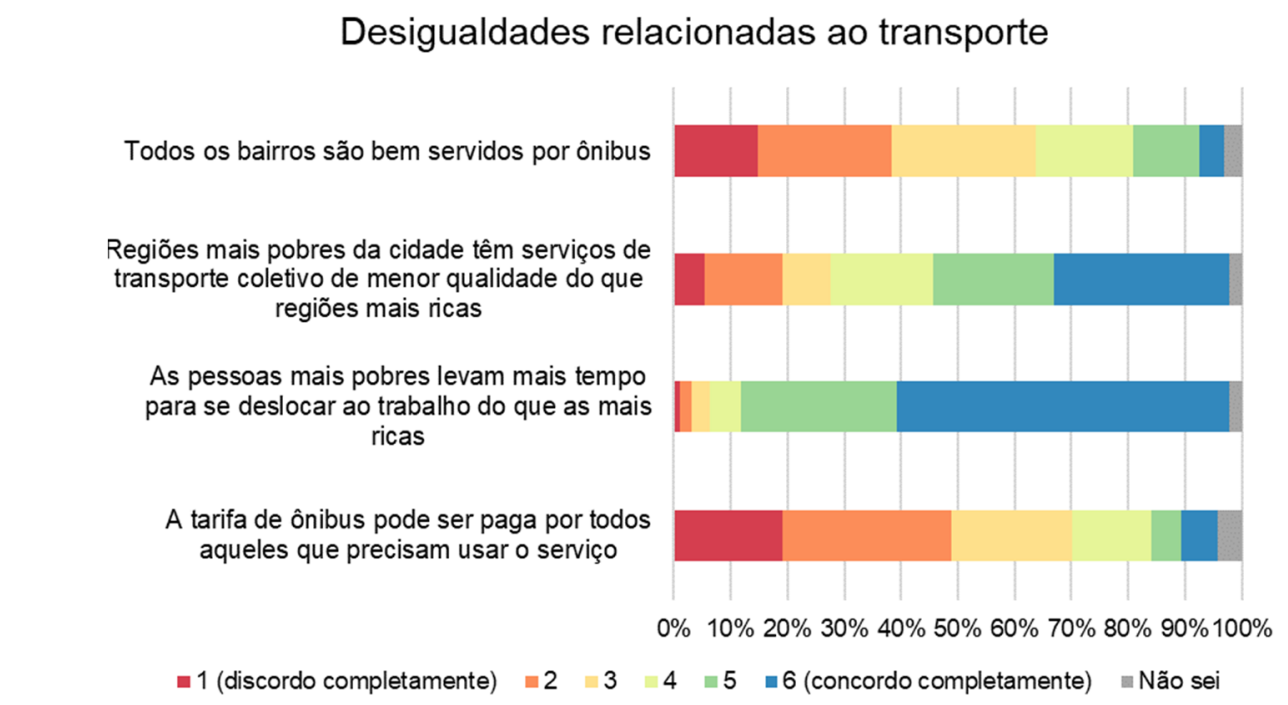

Figura 1. Atitudes dos participantes com relação à existência de desigualdades relacionadas ao transporte

\subsection{Equidade em práticas de planejamento de transportes}

A literatura acadêmica oferece uma gama de hipóteses para a existência de desigualdades como as exploradas na Seção 4.2. Com base na revisão da literatura, solicitou-se aos entrevistados se posicionarem em relação às seguintes afirmações:

- Mobilidade é uma condição indispensável para a participação na sociedade.

- As técnicas de planejamento de transporte beneficiam os segmentos mais favorecidos da população.

- As necessidades de deslocamento dos segmentos mais pobres da população são bem conhecidas pelos planejadores de transporte.

- Intervenções do sistema de transporte beneficiam todos os grupos sociais da mesma maneira.

- As políticas de mobilidade podem produzir desigualdades sociais.

A afirmação de que a mobilidade é condição indispensável para a participação na sociedade - tese central da abordagem da exclusão social relacionada ao transporte - atingiu o mais alto nível de concordância entre os participantes na pesquisa: cerca de $70 \%$ concordaram completamente e apenas $1,1 \%$ discordou totalmente desta afirmação. No entanto, as opiniões dos participantes são consideravelmente polarizadas com relação às demais afirmações. Assim, enquanto $28,7 \%$ dos inquiridos estão plenamente de acordo que as políticas de mobilidade ur- 
bana podem produzir desigualdades sociais, $18,1 \%$ discordaram fortemente disso. De modo semelhante, 13,8\% dos entrevistados estão convencidos de que as intervenções de transporte são socialmente neutras, beneficiando igualmente todos os segmentos sociais, enquanto $20,2 \%$ rejeitam essa ideia. Além disso, enquanto 10,6\% acreditam fortemente que técnicas de planejamento de transporte são viesadas, beneficiando grupos em melhor condição na sociedade, 12,8\% discordaram fortemente dessa afirmação. Por fim, o grau de conscientização da comunidade de planejamento sobre as necessidades de transporte dos grupos sociais mais pobres foi uma questão contestada. 11,7\% acreditam que planejadores conhecem bem tais necessidades, e 10,6\% mantêm a opinião contrária. A Figura 2 ilustra claramente que apenas uma declaração é quase consensual entre os participantes, enquanto as respostas favoráveis e desfavoráveis em relação às demais afirmações apresentam padrões praticamente simétricos.

\section{Princípios de planejamento e desigualdades}

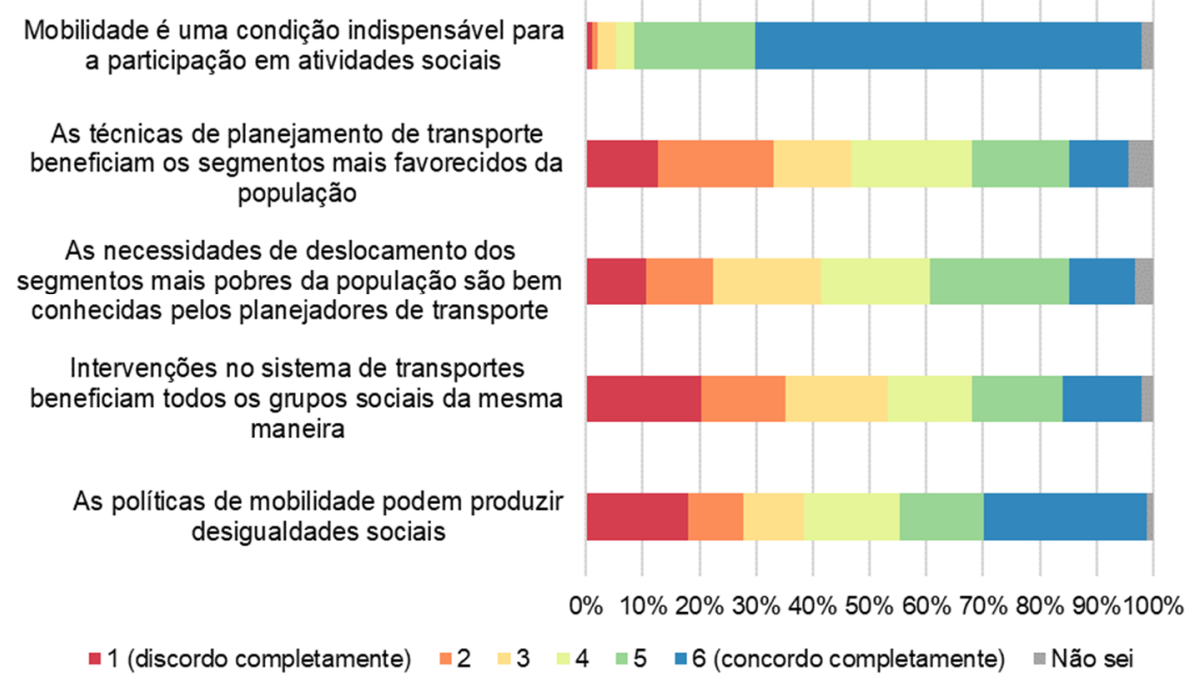

Figura 2. Atitudes dos respondentes com relação a princípios do planejamento de transporte

\subsection{Estratégias prioritárias para o transporte coletivo}

Em seguida, os participantes foram solicitados a selecionar as estratégias mais importantes para aprimorar a qualidade do transporte público urbano por ônibus. Uma lista de 12 estratégias geralmente consideradas por planejadores de transporte público foi apresentada em ordem aleatória, além de um campo para respostas abertas e a opção "Eu não sei". Cada participante foi convidado a selecionar as quatro estratégias que considera ser mais importantes para melhorar os serviços locais de ônibus.

Uma vez que a equidade no transporte é um conceito abrangente e multidimensional, seria inapropriado classificar cada uma das declarações como relacionada ou não ao objetivo de aumentar a equidade. Cada estratégia pode estar relacionada a um aspecto diferente da equidade, dependendo das dimensões consideradas e das circunstâncias concretas em que possa ser aplicada. No entanto, cabe destacar que três das estratégias sugeridas (números 1, 8 e 9) mantêm relação explícita com a equidade social e espacial, tendo sido discutidos com bastante frequência na literatura acadêmica especializada.

A Tabela 1 lista as estratégias elencadas como prioritárias, ordenadas de acordo com a frequência de menções pelos participantes. A estratégia mais citada para melhorar o transporte 
público nas cidades brasileiras é considerada diretamente relacionada à diminuição das desigualdades espaciais e a amenizar efeitos da segregação social, amplamente observada em cidades brasileiras. Mais de metade dos entrevistados $(52,1 \%)$ apontou que intensificar a oferta de transporte em porções mal conectadas do território urbano como sua estratégia preferível para melhorar os serviços coletivos. Esse resultado é consistente com a percepção de que alguns bairros não são bem atendidos pelos serviços de transporte público, conforme descrito na Seção 4.2. Outras duas estratégias, frequentemente mencionadas, focam em melhorar a performance operacional dos ônibus (por exemplo, através de faixas exclusivas) e construir paradas mais informativas e confortáveis. As outras duas estratégias, claramente ligadas a princípios de equidade, relacionadas com o preço da tarifa e a prestação de serviços acessíveis a todos os cidadãos, foram citadas com frequência menor (mencionadas por 30,9\% e 20,2\% dos entrevistados, respectivamente).

Tabela 1 - Estratégias prioritárias para o serviço de ônibus urbano

\begin{tabular}{lll}
\hline & Estratégia & Menções (\%) \\
\hline 1 & Criar ou reorganizar linhas para conectar bairros mal servidos por transporte coletivo & 52,1 \\
2 & Aumentar a velocidade média (p. ex. faixas exclusivas ou corredores) & 50,0 \\
3 & Construir paradas mais informativas e mais confortáveis & 50,0 \\
4 & Aprimorar a integração com outros modos (p. ex. estacionamentos em terminais) & 46,8 \\
5 & $\begin{array}{l}\text { Aprimorar a integração tarifária com outros serviços de transporte coletivo (p. ex. convênios com } \\
\text { municípios vizinhos) }\end{array}$ & 37,2 \\
6 & Aumentar a frequência do serviço (p. ex. mais veículos nas linhas existentes) & 37,2 \\
7 & Renovar a frota de veículos com foco em questões ambientais (p. ex. veículos menos poluentes) & 34,0 \\
8 & Reduzir o preço da tarifa paga & 30,9 \\
9 & Renovar a frota de veículos com foco nas necessidades de pessoas com mobilidade reduzida (p. & 20,2 \\
& ex. veículos com piso rebaixado) & 16,0 \\
10 & Estender o horário em que o serviço é oferecido (p. ex. noite e madrugada) & 11,7 \\
11 & Treinar melhor os motoristas para atender melhor os usuários & 4,3 \\
12 & Combater a informalidade no setor (p. ex. regularizar operadores informais) & 9,6 \\
\hline
\end{tabular}

\subsection{Equidade na mobilidade sustentável}

Finalmente, a pesquisa inquere se a expressão "mobilidade urbana sustentável" é conhecida pelos profissionais e o tipo de projetos a que o conceito está associado. A maioria dos profissionais de planejamento do transporte no Brasil se diz familiarizada com esse conceito. 84,0\% dos respondentes disseram que já lidaram profissionalmente com projetos de mobilidade sustentável e 56,4\% conhecem, na região onde trabalham, ao menos uma intervenção de mobilidade urbana que pode ser considerada sustentável.

Quando solicitados a citar um projeto conhecido de mobilidade urbana sustentável, os participantes mencionaram (em respostas espontâneas) intervenções de diversos tipos, escopos, escalas geográficas e em diferentes estágios de implantação. Após uma análise temática, as respostas foram agrupadas em torno de cinco objetivos gerais, como mostra a Tabela 2.

Em seguida, os participantes foram questionados especificamente sobre seu conhecimento sobre projetos de mobilidade urbana com foco na dimensão social da sustentabilidade, incluindo questões relativas a equidade e justiça social. 54,3\% afirmaram que conhecem um projeto com essa ênfase $(40,4 \%$ podem mencionar um projeto na cidade ou região onde eles trabalham) e novamente as respostas abordaram um amplo espectro de ações e estratégias. No entanto, uma parcela significativa citou o mesmo projeto mencionado na questão anterior, enfatizando 
aspectos que não necessariamente se relacionam com equidade ou em que esta relação não está explícita. As respostas destacaram os aspectos expostos na Tabela 3 que, novamente, puderam ser sintetizados em cinco objetivos gerais.

Tabela 2 - Objetivos dos projetos de mobilidade urbana sustentável citados pelos participantes

\begin{tabular}{ll}
\hline Objetivos gerais & Objetivos específicos \\
\hline 1 Reduzir a emissão de poluentes atmosféricos por & - Introduzir veículos elétricos ou híbridos na frota de transporte \\
meio da adoção de novas tecnologias veiculares & coletivo \\
2 Melhorar a infraestrutura para "modos sustentá- & - Readequar passeios e calçadas \\
veis" & - Ampliar a infraestrutura cicloviária \\
& - Expandir faixas exclusivas de ônibus \\
3 Promover a integração intermodal & - Implementar o Bus Rapid Transit \\
& - Equipar terminais de transporte com paraciclos \\
4 Elaborar e aprovar planos e marcos legais para a & - Oferecer bicicletas públicas a usuários de transporte coletivo \\
mobilidade sustentável & - Desenvolver ou revisar planos cicloviários \\
5 Implementar projetos com foco na mudança de & - Aperfeiçoar instrução normativa federal \\
comportamento & - Retar programas de gestão da mobilidade \\
& - de pedestres \\
& $\begin{array}{l}\text { - Incentivar o compartilhamento de viagens de automóvel por meio de } \\
\text { aplicativo }\end{array}$ \\
\hline
\end{tabular}

Tabela 3 - Objetivos dos projetos de mobilidade urbana socialmente sustentável citados pelos participantes

\begin{tabular}{|c|c|c|}
\hline & Objetivos gerais & Objetivos específicos \\
\hline 1 & $\begin{array}{l}\text { Aumentar a conveniência do uso de meios de } \\
\text { transporte não motorizados }\end{array}$ & $\begin{array}{l}\text { - Implementar planos cicloviários } \\
\text { - Proibir o trânsito de veículos motorizados em certas vias }\end{array}$ \\
\hline 2 & $\begin{array}{l}\text { Fortalecer mecanismos de controle e participa- } \\
\text { ção social das intervenções na mobilidade ur- } \\
\text { bana }\end{array}$ & - Fomentar abordagens participativas no planejamento do transporte \\
\hline 3 & $\begin{array}{l}\text { Expandir a oferta de serviços de transporte cole- } \\
\text { tivo em áreas socioeconomicamente desfavore- } \\
\text { cidas }\end{array}$ & - Introduzir linhas de ônibus e teleféricos em bairros pobres \\
\hline 4 & $\begin{array}{l}\text { Garantir a acessibilidade financeira dos serviços } \\
\text { de transporte a diversos grupos sociais }\end{array}$ & $\begin{array}{l}\text { - Revisar a política tarifária dos meios coletivos } \\
\text { - Aprimorar a integração tarifária } \\
\text { - Conceder gratuidade tarifária para certos segmentos ou para toda } \\
\text { população }\end{array}$ \\
\hline 5 & $\begin{array}{l}\text { Satisfazer necessidades especiais de pessoas } \\
\text { com deficiência ou mobilidade reduzida }\end{array}$ & $\begin{array}{l}\text { - Desenvolver aplicativos de smartphones acessíveis para pessoas com } \\
\text { deficiência visual } \\
\text { - Adaptar a frota de ônibus (piso baixo) }\end{array}$ \\
\hline
\end{tabular}

\section{CONCLUSÕES}

Este estudo procurou elucidar os significados e a importância da equidade no planejamento de transportes no Brasil, com base em evidência empírica obtida por meio de uma pesquisa do tipo survey. Diferentemente do que a literatura sugere, o presente estudo indica que, no Brasil, os profissionais de transportes reconhecem a coexistência de algumas formas de privação socioeconômica (por exemplo, viver em situação de pobreza ou residir em áreas pobres) e privações relacionadas ao transporte (por exemplo, viver em áreas com baixa densidade de serviços de transporte coletivo ou ter acesso direto a serviços de menor qualidade). Ou seja, os profissionais não são desatentos às desigualdades sociais tangentes à segregação residencial urbana, e como estas estão associadas com a oferta e ao uso de transporte. Ao contrário, eles percebem que populações de baixa renda enfrentam, em média, tempos de viagem significativamente maiores para realizar atividades diárias como ir ao trabalho. Também veem o custo tarifário como uma 
severa barreira no acesso aos serviços de transporte e às oportunidades distribuídas nas áreas urbanas. Os profissionais entrevistados entendem ainda que os grupos de baixa renda podem ter suas possibilidades de participação social restritas em função da falta de acesso a serviços de transporte, aumentando o risco de exclusão social.

Talvez por perceber tais desigualdades como injustas, a maioria dos participantes considera que a ampliação dos serviços de transporte coletivo em áreas socialmente desfavorecidas e periféricas deveria receber prioridade. Dessa forma, revelam consciência sobre a necessidade de combater desigualdades relativas à oferta e à cobertura espacial dos serviços, que, no contexto da maioria das cidades brasileiras de médio e grande porte, imbricam-se com o perfil socioeconômico da população usuária. Entretanto, outros aspectos relacionados à equidade fundamentais em uma perspectiva de transporte socialmente inclusivo (por exemplo, preocupações com as necessidades de idosos e de pessoas com mobilidade reduzida) não recebem o mesmo grau de prioridade.

Entretanto, planejadores possuem opiniões divergentes sobre seu papel profissional coletivo como agentes na produção, consolidação ou ampliação de desigualdades relacionadas ao transporte. Uma parte significativa acredita na neutralidade das técnicas de planejamento de transporte empregadas, rejeitando a possibilidade de que certos procedimentos possam produzir resultados socialmente enviesados ou injustos. Mais da metade dos entrevistados discorda que políticas de transporte possam produzir resultados diferentes para grupos sociais distintos ou estar ligadas a desigualdades sociais. Portanto, embora a maioria dos participantes reconheça o papel crucial do transporte para melhores chances de participação na sociedade e perceba a sobreposição entre certas formas de privação social e disparidades na oferta de transporte, eles não entendem essas desigualdades como resultantes do planejamento de transportes. Tais posicionamentos sugerem que fatores externos ao processo de planejamento podem distorcer as intervenções de transporte planejadas.

A pesquisa também revela que, embora o conceito de mobilidade urbana sustentável seja amplamente difundido entre profissionais de planejamento no Brasil, esse conceito não compreende nem imediata nem necessariamente preocupações com a equidade social. Dos projetos mencionados espontaneamente, muitos aludem a necessidade de ampliação da infraestrutura e do favorecimento das condições de circulação de modos não-motorizados de transporte, por exemplo. Tais projetos remetem ao objetivo de aumentar a equidade entre modos de transporte, revertendo a situação muito comum em que viagens por automóvel individual são muito mais convenientes do que por outros modos. No entanto, entre os profissionais há bastante desconhecimento sobre a dimensão social da mobilidade sustentável. Nenhum participante menciona a melhoria da acessibilidade, em particular àquelas atividades essenciais para inclusão social, como objetivo central de um projeto que consideram socialmente sustentável, embora este seja um tópico crucial nos debates acadêmicos contemporâneos sobre equidade em transportes. No geral, os projetos aludidos dizem respeito à capacidade de pagamento pelo uso de sistemas de transporte e ao atendimento de necessidades especiais de pessoas com deficiência e mobilidade reduzida.

Evidências apresentadas por este estudo apontam, assim, para um desalinhamento entre as percepções dos planejadores de transportes e o entendimento corrente na literatura com relação a três tópicos: a relação causal entre a provisão de transporte coletivo urbano e desigualdades socioespaciais; a centralidade da acessibilidade a serviços, equipamentos urbanos e oportunidades essenciais como mecanismo para o bem-estar e a qualidade de vida, em particular de 
populações socioeconomicamente vulneráveis; e a abrangência do conceito de sustentabilidade aplicada à mobilidade urbana, em particular com relação a sua dimensão social, que envolve considerações sobre justiça social.

Certamente, a pesquisa se beneficiaria de um maior tamanho amostral, o que poderia permitir analisar percepções e atitudes estratificadas pela formação acadêmica e região de atuação dos respondentes, por exemplo. Mais importante, no entanto, seria explorar em maior profundidade os resultados deste estudo por meio de técnicas qualitativas, buscando obter explicações alternativas sobre os mecanismos causadores do surgimento e da sustentação de desigualdades sociais associadas a desigualdades em transportes, e refinar a maneira como, no contexto brasileiro, o planejamento poderia lidar com essas questões, no paradigma da "mobilidade sustentável" ou para além dele.

\section{AGRADECIMENTOS}

Os autores agradecem ao Conselho Nacional de Desenvolvimento Científico e Tecnológico (CNPq) e aos revisores anônimos pelos valiosos comentários em versões prévias deste manuscrito.

\section{REFERÊNCIAS}

Arsenio, E.; K. Martens e F. Di Ciommo (2016) Sustainable urban mobility plans: Bridging climate change and equity targets? Research in Transportation Economics, v. 55, p. 30-39. doi:10.1016/j.retrec.2016.04.008

Banister, D. (2002) Transport planning. Spon, Londres.

Banister, D. (2005) Unsustainable transport: City transport in the new century. Routledge, Londres.

Banister, D. (2008) The sustainable mobility paradigm. Transport Policy, v. 15, n. 20, p. 73-80. doi:10.1016/j.tranpol.2007.10.005

Bertolini, L. (2012) Integrating mobility and urban development agendas: A manifesto. disP - The Planning Review, v. 48, n. 1, p. 16-26. doi:10.1080/02513625.2012.702956

Bertolini, L.; F. le Clercq e T. Straatemeier (2008) Urban transportation planning in transition. Transport Policy, v. 15, n. 2, p. 69-72. doi:10.1016/j.tranpol.2007.11.002

Bickerstaff, K.; R. Tolley e G. Walker (2002) Transport planning and participation: The rhetoric and realities of public involvement. Journal of Transport Geography, v. 10, n. 1, p. 61-73. doi:10.1016/S0966-6923(01)00027-8

Bocarejo, J. P. e D. R. Oviedo (2012) Transport accessibility and social inequities: A tool for identification of mobility needs and evaluation of transport investments. Journal of Transport Geography, v. 24, p. 142-154. doi:10.1016/j.jtrangeo.2011.12.004

Boschmann, E. E., e M.-P. Kwan (2008) Toward socially sustainable urban transportation: Progress and potentials. International Journal of Sustainable Transportation, v. 2, n. 3, p. 138-157. doi:10.1080/15568310701517265

Bullard, R. D., e G. S. Johnson (eds.) (1997) Just transportation: Dismantling race and class barriers to mobility. New Society, Gabriola Island.

Caggiani, L.; R. Camporeale e M. Ottomanelli (2017) Facing equity in transportation Network Design Problem: A flexible constraints based model. Transport Policy, v. 55, p. 9-17. doi:10.1016/j.tranpol.2017.01.003

Carruthers, R.; M. Dick e A. Saurkar (2005) Affordability of public transport in developing countries (No. 3). The World Bank, Washington. Disponível em: <https://openknowledge.worldbank.org/handle/10986/17408> (acesso em 18/12/2019).

Cass, N.; E. Shove e J. Urry (2005) Social exclusion, mobility and access. The Sociological Review, v. 53, n. 3, p. 539-555. doi:10.1111/j.1467-954X.2005.00565.x

Chakraborty, J.; J. A. Maantay e J. D. Brender (2011) Disproportionate proximity to environmental health hazards: Methods, models, and measurement. American Journal of Public Health, v. 101, n. S1, p. S27-S36. doi:10.2105/AJPH.2010.300109

Chakraborty, J.; L. A. Schweitzer e D. J. Forkenbrock (1999) Using GIS to assess the environmental justice consequences of transportation system changes. Transactions in GIS, v. 3, n. 3, p. 239-258. doi:10.1111/1467-9671.00020

Church, A.; M. Frost e K. Sullivan (2000) Transport and social exclusion in London. Transport Policy, v. 7, n. 3, p. $195-205$. doi:10.1016/S0967-070X(00)00024-X

Clark, L. P.; D. B. Millet e J. D. Marshall (2017) Changes in transportation-related air pollution exposures by race-ethnicity and socioeconomic status: Outdoor nitrogen dioxide in the United States in 2000 and 2010. Environmental Health Perspectives, v. 125, n. 9, p. 1-10. doi:10.1289/EHP959

Cresswell, T. (2006) The right to mobility: The production of mobility in the courtroom. Antipode, v. 38, n. 4, p. $735-754$. doi:10.1111/j.1467-8330.2006.00474.x

Curl, A.; J. Clark e A. Kearns (2018) Household car adoption and financial distress in deprived urban communities: A case of forced car ownership? Transport Policy, v. 65, p. 61-71. doi:10.1016/j.tranpol.2017.01.002 
Currie, G., e A. Delbosc (2009) Car ownership and low income on the urban fringe-Benefit or hindrance. Proceedings of the 32nd Australasian Transport Research Forum. Disponível em: <http://www.cmnzl.co.nz/assets/sm/4483/61/paper12Currie.pdf> (acesso em 18/12/2019).

Dimitriou, H. T. (2011) Urban transport planning: A developmental approach. Routledge, Londres.

Elvy, J. (2014) Public participation in transport planning amongst the socially excluded: An analysis of 3rd generation local transport plans. Social exclusion in the countries with advanced transport systems, v. 2, n. 2, p. 41-49. doi:10.1016/j.cstp.2014.06.004

Falavigna, C.; T. Guimarães e D. Hernandez (2017a) Mobilidade inclusiva. In: Portugal, L. da S. (ed.) Transporte, mobilidade e desenvolvimento urbano. Elsevier, Rio de Janeiro, p. 219-241.

Falavigna, C.; T. Guimarães e D. Hernandez (2017b) Mobilidade justa socialmente. In: Portugal, L. da S. (ed.) Transporte, mobilidade e desenvolvimento urbano. Elsevier, Rio de Janeiro, p. 243-267.

Falavigna, C. e D. Hernandez (2016) Assessing inequalities on public transport affordability in two Latin American cities: Montevideo (Uruguay) and Córdoba (Argentina). Transport Policy, v. 45, p. 145-155. doi:10.1016/j.tranpol.2015.09.011

Farrington, J. e C. Farrington (2005) Rural accessibility, social inclusion and social justice: Towards conceptualisation. Journal of Transport Geography, v. 13, n. 1, p. 1-12. doi:10.1016/j.jtrangeo.2004.10.002

Forkenbrock, D.; S. Benshoff e G. Weisbrod (2001) Assessing the social and economic effects of transportation projects (NCHRP Web Document No. 31). Disponível em: <http://onlinepubs.trb.org/onlinepubs/nchrp/nchrp_w31.pdf> (acesso em 18/12/2019).

Fouracre, P. R.; M. Sohail e S. Cavill (2006) A participatory approach to urban transport planning in developing countries. Transportation Planning and Technology, v. 29, n. 4, p. 313-330. doi:10.1080/03081060600905665

Gaffron, P. (2012) Urban transport, environmental justice and human daily activity patterns. Transport Policy, v. 20, p. 114127. doi:10.1016/j.tranpol.2012.01.011

Geurs, K. T.; W. Boon, e B. Van Wee (2009) Social impacts of transport: Literature review and the state of the practice of transport appraisal in the Netherlands and the United Kingdom. Transport Reviews, v. 29, n. 1, p. 69-90. doi:10.1080/01441640802130490

Godard, X. (2011) Poverty and urban mobility: Diagnosis toward a new understanding. In: Dimitriou, H. e R. A. Garkenheimer (eds.) Urban transport in the developing world: A handbook of policy and practice. Edward Elgar, Cheltenham, p. $232-261$.

Grieco, M. (2015) Social sustainability and urban mobility: Shifting to a socially responsible pro-poor perspective. Social Responsibility Journal, v. 11, n. 1, p. 82-97. doi:10.1108/SRJ-05-2014-0061

Gudmundsson, H.; R. P. Hall; G. Marsden e J. Zietsman (2016) Transportation and sustainability. In: Gudmundsson, H.; R. P. Hall; G. Marsden e J. Zietsman (eds.) Sustainable transportation: Indicators, frameworks, and performance management. Springer, Berlim, p. 81-109.

Guzman, L. A. e D. Oviedo (2018) Accessibility, affordability and equity: Assessing 'pro-poor' public transport subsidies in Bogotá. Transport Policy, v. 68, p. 37-51. doi:10.1016/j.tranpol.2018.04.012

Guzman, L. A.; D. Oviedo e C. Rivera (2017) Assessing equity in transport accessibility to work and study: The Bogotá region. Journal of Transport Geography, v. 58, p. 236-246. doi:10.1016/j.jtrangeo.2016.12.016

Hananel, R. e J. Berechman (2016) Justice and transportation decision-making: The capabilities approach. Transport Policy, v. 49, p. 78-85. doi:10.1016/j.tranpol.2016.04.005

Hay, A. e E. Trinder (1991) Concepts of equity, fairness, and justice expressed by local transport policymakers. Environment and Planning C: Government and Policy, v. 9, n. 4, p. 453-465. doi:10.1068/c090453

Hine, J. e M. Grieco (2003) Scatters and clusters in time and space: Implications for delivering integrated and inclusive transport. Transport Policy, v. 10, n. 4, p. 299-306. doi:10.1016/S0967-070X(03)00055-6

Hine, J. e F. Mitchell (2001) The role of transport in social exclusion in urban Scotland. Scottish Executive Central Research Unit. Disponível em: < https://www2.gov.scot/resource/doc/156591/0042062.pdf> (acesso em 18/12/2019).

Hodgson, F. C. e J. Turner (2003) Participation not consumption: The need for new participatory practices to address transport and social exclusion. Transport Policy, v. 10, n. 4, p. 265-272. doi:10.1016/j.tranpol.2003.08.001

Houston, D.; J. Wu; P. Ong e A. Winer (2004) Structural disparities of urban traffic in Southern California: Implications for vehicle-related air pollution exposure in minority and high-poverty neighborhoods. Journal of Urban Affairs, 26(5), 565-592. doi:10.1111/j.0735-2166.2004.00215.x

Hull, A. (2008) Policy integration: What will it take to achieve more sustainable transport solutions in cities? Transport Policy, v. 15, n. 2, p. 94-103. doi:10.1016/j.tranpol.2007.10.004

Jaramillo, C.; C. Lizárraga e A. L. Grindlay (2012) Spatial disparity in transport social needs and public transport provision in Santiago de Cali (Colombia). Journal of Transport Geography, v. 24, n. 0, p. 340-357. doi:10.1016/j.jtrangeo.2012.04.014

Jones, P. (2014) The evolution of urban mobility: The interplay of academic and policy perspectives. IATSS Research, v. 38, n. 1, p. 7-13. doi:10.1016/j.iatssr.2014.06.001

Jones, P. e K. Lucas (2012) The social consequences of transport decision-making: Clarifying concepts, synthesising knowledge and assessing implications. Journal of Transport Geography, v. 21, n. 0, p. 4-16. doi:10.1016/j.jtrangeo.2012.01.012

Kenyon, S.; G. Lyons e J. Rafferty (2002) Transport and social exclusion: Investigating the possibility of promoting inclusion through virtual mobility. Journal of Transport Geography, v. 10, n. 3, p. 207-219. doi:10.1016/S0966-6923(02)00012-1

Levinson, D. (2010) Equity effects of road pricing: A review. Transport Reviews, v. 30, n. 1, p. 33-57. doi:10.1080/01441640903189304 
Li, X.; D. Rey e V. V. Dixit (2018) An axiomatic characterization of fairness in transport networks: Application to road pricing and spatial equity. Transport Policy, v. 68, p. 142-157. doi:10.1016/j.tranpol.2018.05.003

Litman, T. (2013) The new transportation planning paradigm. ITE Journal, v. 83, p. 20-28.

Liu, Q.; K. Lucas; G. Marsden e Y. Liu (2019) Egalitarianism and public perception of social inequities: A case study of Beijing congestion charge. Transport Policy, v. 74, p. 47-62. doi:10.1016/j.tranpol.2018.11.012

Lucas, K. (2004) Transport and social exclusion. Running on empty: Transport, social exclusion and environmental justice. Policy Press, Bristol, p. 39-53.

Lucas, K. (2012) A critical assessment of accessibility planning for social inclusion. In: Geurs, K; K. Krizek e A. Reggiani (eds.) Accessibility analysis and transport planning: Challenges for Europe and North America. Edward Elgar, Cheltenham, p. 228242.

Lucas, K.; G. Mattioli; E. Verlinghieri e A. Guzman (2016) Transport poverty and its adverse social consequences. Proceedings of the Institution of Civil Engineers - Transport, v. 169, n. 6, p. 353-365. doi:10.1680/jtran.15.00073

Lucas, K. e J. Stanley (2013) Achieving socially sustainable transport in the development context. Anais da 13th World Conference on Transport Research, Rio de Janeiro. Disponível em <http://www.wctrs-society.com/wp/wp-content/uploads/abstracts/rio/selected/969.pdf> (acesso em 18/12/2019).

Lucas, K.; B. Van Wee e K. Maat (2016) A method to evaluate equitable accessibility: Combining ethical theories and accessibility-based approaches. Transportation, v. 43, n. 3, p. 473-490. doi:10.1007/s11116-015-9585-2

Maia, M. L.; K. Lucas; G. Marinho; E. Santos e J. H. de Lima (2016) Access to the Brazilian City-From the perspectives of lowincome residents in Recife. Journal of Transport Geography, v. 55, p. 132-141. doi:10.1016/j.jtrangeo.2016.01.001

Manaugh, K.; M. G. Badami e A. M. El-Geneidy (2015) Integrating social equity into urban transportation planning: A critical evaluation of equity objectives and measures in transportation plans in North America. Transport Policy, v. 37, p. $167-176$. doi:10.1016/j.tranpol.2014.09.013

Manzi, T.; K. Lucas; T. L. Jones e J. Allen (2010) Understanding social sustainability: Key concepts and developments in theory and practice. In: Manzi, T; K. Lucas; T. L. Jones e J. Allen (eds.) Social sustainability in urban areas: Communities, connectivity and the urban fabric. Earthscan, Londres, p. 1-28.

Markovich, J. e K. Lucas (2011) The social and distributional impacts of transport: a literature review. Transport Studies Unit, School of Geography and the Environment Working Paper, (1055). Disponível em: <http://www.tsu.ox.ac.uk/pubs/1055markovich-lucas.pdf> (acessado em 18/12/2019).

Martens, K. (2012) Justice in transport as justice in accessibility: Applying Walzer's 'Spheres of Justice' to the transport sector. Transportation, v. 39, n. 6, p. 1035-1054. doi:10.1007/s11116-012-9388-7

Martens, K. (2017) Transport justice: Designing fair transportation systems. Routledge, Nova York.

Martens, K., e K. Lucas (2018) Perspectives on transport and social justice. In: G. Craig (ed.) Handbook on global social justice. Edward Elgar, Northampton, p. 351-370.

Mattioli, G. (2015) Energy-related economic stress at the interface between transport, housing and fuel poverty: A multinational study. Université François-Rabelais.

Mattioli, G. (2017) "Forced car ownership" in the UK and Germany: Socio-spatial patterns and potential economic stress impacts. Social Inclusion, v. 5, n. 4, p. 147-160. doi:10.17645/si.v5i4.1081

Mattioli, G.; K. Lucas e G. Marsden (2017) Transport poverty and fuel poverty in the UK: From analogy to comparison. Transport Policy, v. 59, p. 93-105. doi:10.1016/j.tranpol.2017.07.007

Mattioli, G.; Z. Wadud e K. Lucas (2016) Developing a novel approach for assessing the transport vulnerability to fuel price rises at the household level. 14th World Conference on Transport Research. Shanghai.

Milojevic, A.; C. L. Niedzwiedz; J. Pearce; J. Milner; I. A. MacKenzie; R.M. Doherty e P. Wilkinson (2017) Socioeconomic and urban-rural differentials in exposure to air pollution and mortality burden in England. Environmental Health: A Global Access Science Source, v. 16, n. 1. doi:10.1186/s12940-017-0314-5

Ministério das Cidades. (2015) PlanMob - Caderno de referência para elaboração de Plano de Mobilidade Urbana. Disponível em: <http://www.cidades.gov.br/images/stories/ArquivosSE/planmob.pdf> (acesso em 18/12/2019).

Miranda, H. de F. e A. N. Rodrigues da Silva (2012) Benchmarking sustainable urban mobility: The case of Curitiba, Brazil. Transport Policy, v. 21, p. 141-151. doi:10.1016/j.tranpol.2012.03.009

Morency, P.; L. Gauvin; C. Plante; M. Fournier e C. Morency (2012) Neighborhood social inequalities in road traffic injuries: the influence of traffic volume and road design. American Journal of Public Health, v. 102, n. 6, p. 1112-1119. doi:10.2105/AJPH.2011.300528

Mueller, N.; D. Rojas-Rueda; H. Khreis; M. Cirach; C. Milà; A. Espinosa; M. Foraster; R. R. C. McEachan; B. Kelly; J. Wright e M. Nieuwenhuijsen (2018) Socioeconomic inequalities in urban and transport planning related exposures and mortality: A health impact assessment study for Bradford, UK. Environment International, v. 121, p. 931-941. doi:10.1016/j.envint.2018.10.017

Nahmias-Biran, B.-H.; K. Martens e Y. Shiftan (2017) Integrating equity in transportation project assessment: A philosophical exploration and its practical implications. Transport Reviews, v. 37, n. 2, p. 192-210. doi:10.1080/01441647.2017.1276604

Ohnmacht, T.; H. Maksim e M. Bergman (2009) Mobilities and inequalities - Making the connections. T. Ohnmacht, H. Maksim, \& M. M. Bergman (Eds), Mobilities and inequality (p. 7-25). Ashgate Publishing Company, Farnham, England ; Burlington, VT. 
Oviedo Hernandez, D. e H. Titheridge (2016) Mobilities of the periphery: Informality, access and social exclusion in the urban fringe in Colombia. Journal of Transport Geography, v. 55, p. 152-164. doi:10.1016/j.jtrangeo.2015.12.004

Pereira, R. H. M.; D. Banister; T. Schwanen e N. Wessel (2017) Distributional effects of transport policies on inequalities in access to opportunities in Rio de Janeiro (SSRN Scholarly Paper No. ID 3040844). Social Science Research Network, Rochester. Disponível em <https://papers.ssrn.com/abstract=3040844> (acesso em 18/12/2019).

Pereira, R. H. M.; T. Schwanen e D. Banister (2017) Distributive justice and equity in transportation. Transport Reviews, v. 37, n. 2, p. 170-191. doi:10.1080/01441647.2016.1257660

Portugal, L. da S. (2017) Transporte, mobilidade e desenvolvimento urbano. Elsevier, Rio de Janeiro.

Rodrigues da Silva, A. N.; M. da Silva Costa e M. H. Macedo (2008) Multiple views of sustainable urban mobility: The case of Brazil. Decision-support for sustainable urban transport strategies, v. 15, n. 6, p. 350-360. doi:10.1016/j.tranpol.2008.12.003

Rowangould, D.; A. Karner e J. London (2016) Identifying environmental justice communities for transportation analysis. Transportation Research Part A: Policy and Practice, v. 88, p. 151-162. doi:10.1016/j.tra.2016.04.002

Ryan, J.; A. Wretstrand e S. M. Schmidt (2015) Exploring public transport as an element of older persons' mobility: A Capability Approach perspective. Journal of Transport Geography, v. 48, p. 105-114. doi:10.1016/j.jtrangeo.2015.08.016

Sagaris, L. (2010) From sustainable transport development to active citizenship and participatory democracy: The experience of Living City in Chile. Natural Resources Forum, v. 34, n. 4, p. 275-288. doi:10.1111/j.1477-8947.2010.01312.x

Sanchez, T. W. (2008) Poverty, policy, and public transportation. Transportation Research Part A: Policy and Practice, v. 42, n. 5 , p. 833-841. doi:10.1016/j.tra.2008.01.011

Sanchez, T. W.; R. Stolz e J. S. Ma (2003) Moving to equity: Addressing inequitable effects of transportation policies on minorities. Disponível em: <http://escholarship.org/uc/item/5qc7w8qp.pdf> (acesso em 18/12/2019)

Santos, B.; A. Antunes e E. J. Miller (2008) Integrating equity objectives in a road network design model. Transportation Research Record, v. 2089, n. 1, p. 35-42. doi:10.3141/2089-05

Sider, T.; M. Hatzopoulou; N. Eluru; G. Goulet-Langlois e K. Manaugh (2015) Smog and socioeconomics: an evaluation of equity in traffic-related air pollution generation and exposure. Environment and Planning B: Planning and Design, v. 42, n. 5, p. 870-887. doi:10.1068/b130140p

Smith, N.; D. Hirsch e A. Davis (2012) Accessibility and capability: The minimum transport needs and costs of rural households. Journal of Transport Geography, v. 21, p. 93-101. doi:10.1016/j.jtrangeo.2012.01.004

Soja, E. W. (2010) Seeking spatial justice. University of Minnesota Press, Minneapolis.

Thomopoulos, N.; S. Grant-Muller e M. Tight (2009) Incorporating equity considerations in transport infrastructure evaluation: Current practice and a proposed methodology. Evaluation and Program Planning, v. 32, n. 4, p. 351-359. doi:10.1016/j.evalprogplan.2009.06.013

Tonne, C.; C. Milà; D. Fecht; M. Alvarez; J. Gulliver; J. Smith; S. Beevers; H. R. Anderson e F. Kelly (2018) Socioeconomic and ethnic inequalities in exposure to air and noise pollution in London. Environment International, v. 115, p. 170-179. doi:10.1016/j.envint.2018.03.023

Trinder, E.; A. Hay; J. Dignan; P. Else e J. Skorupski (1991) Concepts of equity, fairness, and justice in British transport legislation, 1960-88. Environment and Planning C: Government and Policy, v. 9, n. 1, p. 31-50. doi:10.1068/c090031

Uteng, T. (2008) Gendered mobilities. Ashgate, Aldershot.

Uteng, T. P. (2009) Gender, ethnicity, and constrained mobility: Insights into the resultant social exclusion. Environment and planning. A, v. 41, n. 5, p. 1055. doi:10.1068/a40254

Van Wee, B. (2011) Transport and ethics: ethics and the evaluation of transport policies and projects. Edward Elgar, Londres.

Vasconcellos, E. A. (2001) Urban transport, environment and equity: The case for developing countries. Earthscan, Londres.

Vasconcellos, E. A. (2014) Políticas de transporte no Brasil: A construção da mobilidade excludente. Manole, Barueri.

Vasconcellos, E. A. (2018) Urban transport policies in Brazil: The creation of a discriminatory mobility system. Journal of Transport Geography, v. 67, p. 85-91. doi:10.1016/j.jtrangeo.2017.08.014

Vigar, G. (2006) Deliberation, participation and learning in the development of regional strategies: Transport policy making in North East England. Planning Theory \& Practice, v. 7, n. 3, p. 267-287. doi:10.1080/14649350600841446

Vigar, G. (2017) The four knowledges of transport planning: Enacting a more communicative, trans-disciplinary policy and decision-making. Transport Policy, v. 58, p. 39-45. doi:10.1016/j.tranpol.2017.04.013

Villaça, F. e S. Zioni. (2010) A expansão do metrô de São Paulo: Acentuando desigualdades. Anais do XVI Congresso Pan-Americano de Engenharia de Tráfego e Transportes e Logística (PANAM), Lisboa. 